\title{
TENDÊNCIAS DOS ÍNDICES DE PRECIPITAÇÃO NO ESTADO DO CEARÁ
}

\author{
CARLOS ANTONIO COSTA DOS SANTOS, JOSÉ IVALDO BARBOSA DE BRITO, TANTRAVAHI \\ VENKATA RAMANA RAO, HUDSON ELLEN ALENCAR MENEZES
}

\author{
Universidade Federal de Campina Grande - UFCG, Campina Grande - PB - Brasil.
}

carlostorm@gmail.com, ivaldo@dca.ufcg.edu.br, ramana@dca.ufcg.edu.br, hudsonellen@bol.com.br,

Recebido Fevereiro 2008 - Aceito Novembro 2008

\section{RESUMO}

O principal objetivo deste trabalho é prover novas informações sobre as tendências na precipitação total e eventos extremos de precipitação sobre o Estado do Ceará, fazendo uma análise de diferentes índices de detecção de mudanças climáticas, baseados em dados de precipitação pluvial diária. Utilizaram-se dados de precipitação de 18 postos, no período de 1935 a 2006, representando todas as microrregiões do Estado; o software utilizado no processamento e controle de qualidade dos dados foi o RClimdex 1.9.0. Com base nos resultados encontrados, observou-se que ocorreram mudanças locais na precipitação, assim como um visível aumento nas condições de umidade sobre o Ceará.

Palavras Chaves: Mudanças Climáticas, Semi-árido, RClimdex 1.9.0

\begin{abstract}
TRENDS OF PRECIPITATION INDICES IN CEARÁ STATE
The principal objective of this work is to provide new information on the trends in the total precipitation and extreme events of precipitation in the State of Ceará, making an analysis of different indices of detection of climatic changes, based on data of daily precipitation. Precipitation data of 18 stations were used, in the period from 1935 to 2006, representing all the microregions of the State; the software used in the processing and quality control of the data was RClimdex 1.9.0. The results show local changes in the precipitation and a visible increase in the humidity conditions in Ceará.
\end{abstract}

Keywords: Climate Change, Semi-arid, RClimdex 1.9.0

\section{INTRODUÇÃO}

As mudanças no clima podem acarretar significativos impactos nos setores naturais, social e econômico. Os extremos climáticos associados à temperatura e precipitação podem também afetar consumo de energia, conforto humano e turismo (Subak et al., 2000; Qian e Lin, 2005). O aumento nas perdas econômicas devido a extremos de tempo e, especialmente, o aumento nas perdas de vidas, tem sido regularmente notícias de jornais, despertando o interesse da comunidade científica em estudar tais fenômenos (Kostopoulo e Jones, 2005).

Mudanças na precipitação têm implicações no ciclo hidrológico e recursos hídricos. Espera-se que as mudanças climáticas alterem a temperatura média e os valores da precipitação e que aumentem a variabilidade dos eventos de precipitação, que poderão causar inundações e secas mais intensas e freqüentes. Qian e Lin (2005) afirmam que a freqüência e a persistência das secas deverá ser uma das conseqüências do aquecimento global. Walsh e Pittock (1998) sugeriram que as potenciais mudanças nas tempestades tropicais, furacões e eventos extremos de chuva são um resultado das mudanças climáticas. Em geral, muitas atividades econômicas e processos ambientais são altamente dependentes da precipitação. No Nordeste do Brasil (NEB), as atividades agrícolas, quase que em sua totalidade, são baseadas na precipitação e o conhecimento de sua variabilidade é de extrema importância.

De forma geral, a atmosfera é constituída por vários gases, sendo que os principais são o Nitrogênio $\left(\mathrm{N}_{2}\right)$ e o Oxigênio $\left(\mathrm{O}_{2}\right)$ que, juntos compõem cerca de $99 \%$ da proporção em volume. Outros gases encontram-se presentes em pequenas quantidades, 
dentre estes, citam-se os gases de efeito estufa (GEE) tais como o dióxido de carbono $\left(\mathrm{CO}_{2}\right)$. São assim denominados por ter a capacidade natural de reter o calor na atmosfera, permitindo que as ondas eletromagnéticas provenientes do Sol atravessem a atmosfera e aqueçam a superfície terrestre, mas dificultando a saída da radiação infravermelha emitida pela Terra, mantendo, assim a Terra aquecida.

Nos últimos 100 anos vem acontecendo um progressivo aumento na concentração dos GEE na atmosfera, sendo provocado, principalmente pelas atividades antrópicas (IPCC, 2007). Segundo o Relatório do IPCC (2007), para as próximas duas décadas, projeta-se um aquecimento de cerca de $0,2^{\circ} \mathrm{C}$ por década para uma faixa de cenários de emissões do RECE (Relatório Especial sobre Cenários de Emissões). Mesmo que as concentrações de todos os gases de efeito estufa e aerossóis se mantivessem constantes nos níveis do ano 2000, seria esperado um aquecimento adicional de cerca de $0,1^{\circ} \mathrm{C}$ por década. Até meados do século XXI, projeta-se que a vegetação semi-árida tenderá a ser substituída por vegetação de terras áridas. Nas áreas mais secas, prevê-se que a mudança do clima acarrete a salinização e a desertificação das terras agrícolas. Projeta-se que as mudanças nos padrões de precipitação e o desaparecimento das geleiras afetem de forma significativa a disponibilidade de água para o consumo humano, a agricultura e a geração de energia (IPCC, 2007).
Na América Latina, tem-se observado uma grande variedade de alterações no clima, que podem ser resultantes não só da variabilidade climática natural como também da interferência humana no sistema. Notam-se também, variações principalmente no ciclo hidrológico e na temperatura média (IPCC, 2007). Pesquisadores como Hooper et al (2005) afirmam que fatores antropicos, tais como: desflorestamento e mau uso dos ecossistemas, podem modificar o clima de uma região. Esses fatores alteram o clima regional contribuindo para as alterações no sistema global. Em áreas de ecossistemas frágeis e vulneráveis, tais como o Semi-árido Brasileiro, as mudanças climáticas mais drásticas poderão ocorrer através da soma das ações produzidas pelos GEE com o mau uso e desflorestamento dos ecossistemas locais (Santos e Brito, 2007).

Objetivando identificar mudanças climáticas regionais, recentemente, Haylock et al. (2006) fizeram uma análise da precipitação sobre a América do Sul, e observaram uma tendência de aumento do total anual de chuva sobre o NEB. O estudo realizado por Santos e Brito (2007), utilizando índices de extremos climáticos e correlacionando-os com as anomalias de temperatura da superfície do mar (TSM), também mostra tendência de aumento da precipitação total anual nos estados da Paraíba e Rio Grande do Norte.

Vale salientar que, em geral, as mudanças climáticas locais são medidas por meio de análise de séries históricas

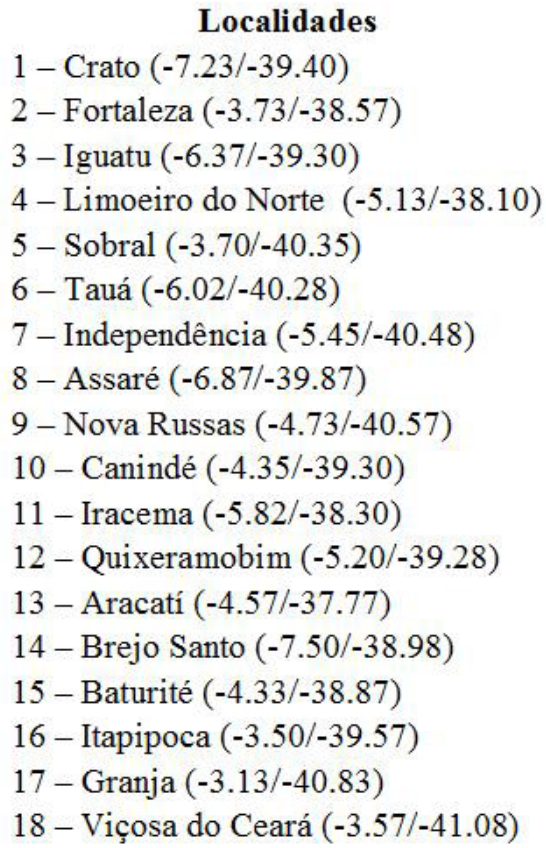

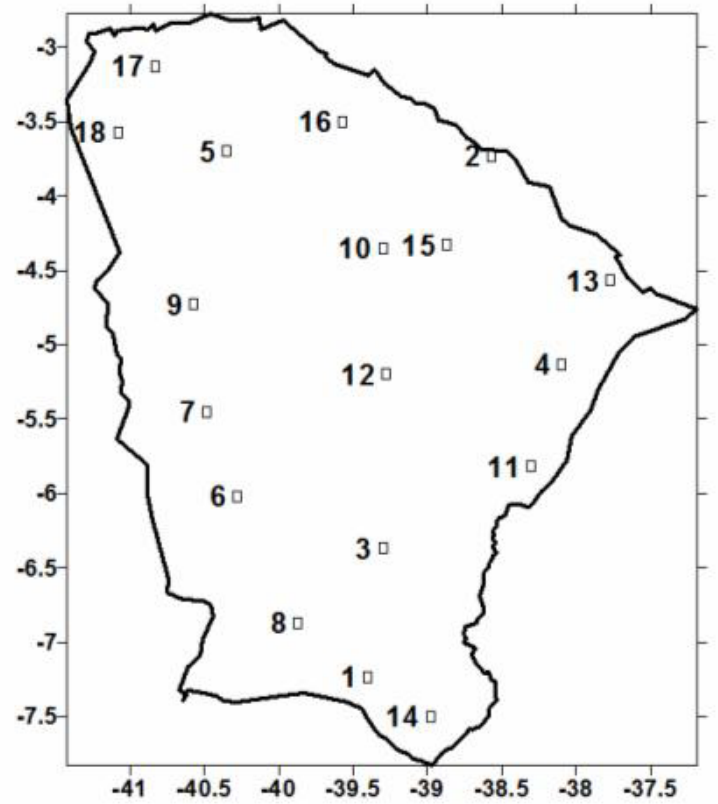

Figura 1 - Distribuição espacial das 18 localidades escolhidas no Estado do Ceará, com os seus respectivos números identificadores e coordenadas geográficas. 
de variáveis meteorológicas, tais como, temperatura do ar e precipitação pluvial. De acordo com as tendências temporais dessas variáveis para um determinado local é observado se ocorreram mudanças no clima. Entretanto, para melhor definir essa questão a Organização Meteorológica Mundial (OMM) criou um grupo de trabalho que elaborou 27 índices de detecção de mudanças climáticas, sendo que 11 são decorrentes da precipitação e 16 da temperatura do ar (Zhang e Yang, 2004).

A precipitação é uma variável determinante das condições do clima no semi-árido brasileiro, bem como da sua variabilidade e mudança a longo prazo. Portanto, nesse contexto este trabalho tenta prover novas informações de tendências na precipitação total e eventos extremos de precipitação sobre o Estado do Ceará, localizado no NEB, através da análise de diferentes índices de detecção de mudanças climáticas, baseados em dados observacionais de várias estações na região. $\mathrm{O}$ foco desse trabalho é particularmente importante para o Estado do Ceará, uma vez que alguma mudança no clima poderá ter profundas influências sócio-econômicas, e consequentemente no bem-estar da população, como também causar impactos ambientais.

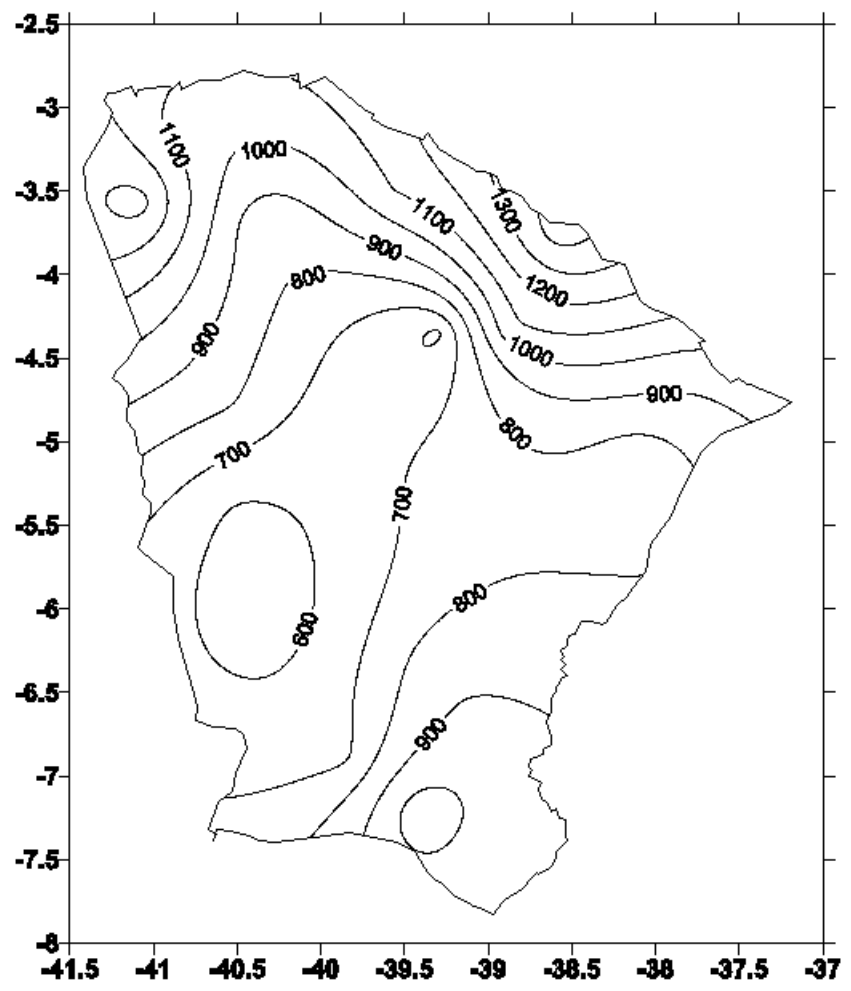

Figura 2 - Distribuição espacial da precipitação média anual, em $\mathrm{mm} / \mathrm{ano}$.

\section{DADOS E METODOLOGIA}

Os dados de precipitação diária foram oriundos da antiga rede de postos pluviométricos da Superintendência de Desenvolvimento do Nordeste (SUDENE) e da Fundação Cearense de Meteorologia (FUNCEME). Entre 50 postos pluviométricos disponíveis, foram escolhidos 18 postos, que apresentaram dados de 1935 a 2006, com poucas falhas, e representam todas as microrregiões do Estado (Figura 1). O controle de qualidade dos dados inclui a consistência espacial e temporal dos valores diários da precipitação. Para minimizar as influências associadas com a heterogeneidade temporal, foram removidas as estações com sérios eventos de relocação e séries com dados errôneos e unidades incorretas.

Na Tabela 1, estão apresentados os seis índices climáticos, derivados da precipitação pluvial, utilizados nessa pesquisa, conforme a definição realizada pelo "Expert Team on Climate Change Detection Monitoring and Indices (ETCCDMI)". Na avaliação da homogeneização dos dados e no cálculo dos índices foi utilizado a metodologia de Zhang et al. (2005) e Haylock et al. (2006), em que utilizou-se o software RClimdex 1.9.0,

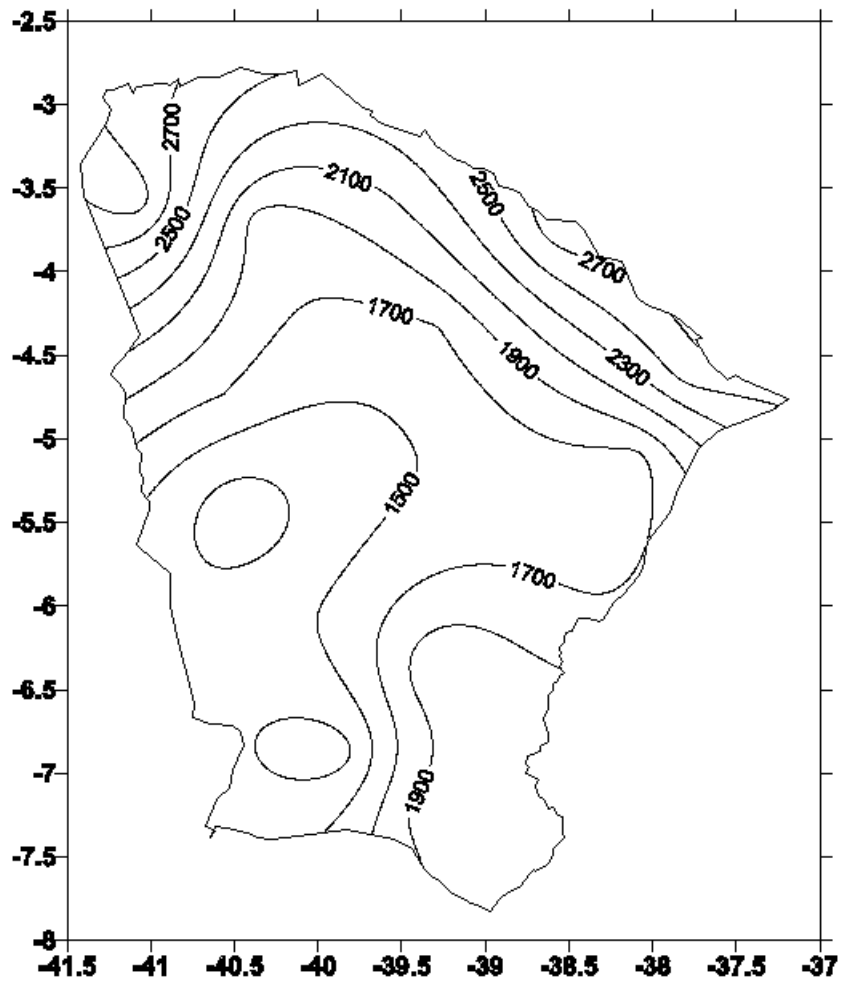

Figura 3 - Distribuição espacial da precipitação máxima, em mm/ ano. 
desenvolvido e mantido pelos pesquisadores Xuebin Zhang e Feng Yang do Serviço de Meteorologia do Canadá. Este software está disponível gratuitamente no site http://cccma.seos.uvic. ca/ETCCDMI/software.html. Para sua execução é necessário seguir os seguintes passos: (1) dispor os dados em arquivo de texto ASCII, composto de seis colunas correspondentes ao ano, mês, dia, precipitação (PRCP), temperatura máxima (TMAX) e temperatura mínima (TMIN); (2) os dados faltosos são codificados como -99.9 e os registros dos dados dispostos em ordem cronológica (CANADIAN INTERNATIONAL DEVELOPMENT AGENCY, 2004).

O principal propósito do controle de qualidade foi identificar erros no processamento dos dados, assim como erros de coleta manual dos mesmos, conforme proposto por Alexander et al. (2006). Foram feitos testes estatísticos e uma investigação do histórico das estações ou comparações com estações vizinhas para determinar se os valores da precipitação eram corretos, conforme metodologia proposta por Viney e Bates (2004).

O RClimdex 1.9.0 fornece, para todos os índices, dados estatísticos, tais como: tendência linear calculada pelo método de mínimos quadrados; nível de significância estatística da tendência (valor $p$ ); coeficiente de determinação $\left(r^{2}\right)$ e erro

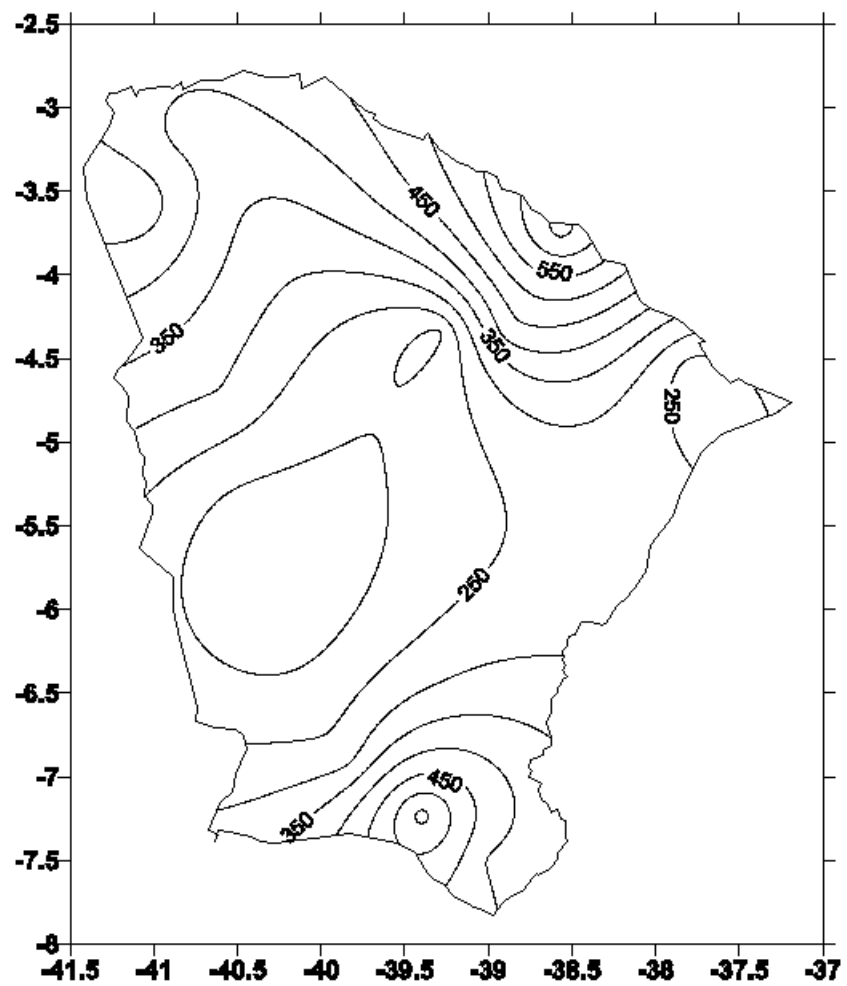

Figura 4 - Distribuição espacial da precipitação mínima, em mm/ ano. padrão de estimativa; assim como os gráficos das séries anuais. Sendo assim, foram consideradas como possíveis mudanças climáticas aquelas em que o índice apresentava tendência linear (positiva ou negativa) superior ao erro padrão de estimativa e estatisticamente significativa (valor de $\mathrm{p}<0.1$ ).

A representação, na distribuição espacial, das tendências dos seis índices foi feita utilizando o símbolo (+), para as tendências positivas, e $(\bullet)$ para as negativas, estatisticamente significantes, ou seja, que apresentaram o valor de $\mathrm{p}<0,1$. Para as que não apresentaram significância estatística, utilizaram-se os símbolos $(+)$, para as positivas, e $(\mathrm{O})$, para as negativas. $\mathrm{O}$ tamanho do símbolo representa a magnitude da tendência, ou seja, quanto maior o símbolo maior a tendência e vice e versa. No entanto, o tamanho do símbolo não é exatamente proporcional aos valores das mesmas, e sim, uma tentativa de tornar visível o comportamento das referidas tendências.

\section{RESULTADOS}

Para melhor entender a contribuição dos índices extremos de precipitação para o clima do Estado do Ceará, a climatologia da precipitação está mostrada a seguir. A Figura 2 mostra a

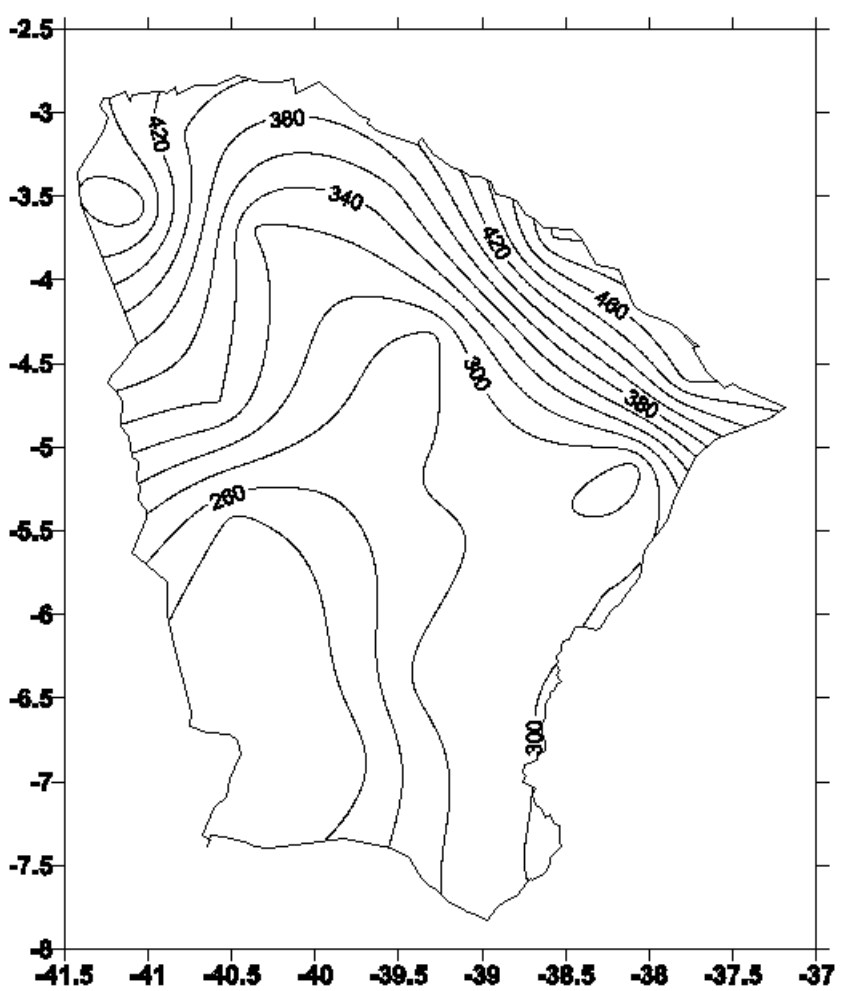

Figura 5 - Distribuição espacial do desvio padrão da precipitação, em mm/ano. 
distribuição espacial da precipitação média anual, com valores variando de $1400 \mathrm{~mm}$, no litoral a valores inferiores a $600 \mathrm{~mm}$, no sertão. $\mathrm{O}$ padrão da distribuição da precipitação máxima (Figura 3) e mínima (Figura 4) foi similar ao da precipitação média (Figura 2). Na Figura 3 pode-se observar que os valores variam de $2700 \mathrm{~mm}$ no litoral, a $1400 \mathrm{~mm}$ no sertão. Na Figura 4 observa-se valores da ordem de $600 \mathrm{~mm}$ no litoral e inferiores a $250 \mathrm{~mm}$ no sertão. A Figura 5 apresenta a distribuição espacial do desvio padrão da precipitação anual, identificando-se valores superiores a $460 \mathrm{~mm}$ no litoral e inferiores a $260 \mathrm{~mm}$ no sertão, mostrando assim, a grande variabilidade da precipitação no Estado.

Os valores das tendências temporais, dos índices de extremos climáticos, estão apresentados na Tabela 2, para todas as 18 localidades. Os valores realçados e em negrito apresentaram alta significância estatística $(\mathrm{p} \leq 0,05)$, os apenas realçados apresentaram boa significância estatística $(0,05<\mathrm{p} \leq$ $0,1)$ e para os demais valores, o $\mathrm{p}$ estimado foi superior a 0,1 .

Analisando a Tabela 2 observam-se tendências, estatisticamente significantes, de mudanças dos Dias Consecutivos Secos (DCS) em 5 das localidades e dos Dias Consecutivos Úmidos (DCU) em 6 localidades. No caso de DCS, das 5 localidades 4 delas apresentaram aumento, enquanto para o índice DCU ocorreram 5 tendências de aumento. No entanto, das localidades que apresentaram significância estatística no DCS apenas Nova Russas apresentou em DCU, demonstrando que para essa localidade, ocorreu um aumento no número de dias consecutivos secos e uma diminuição dos dias consecutivos com chuvas. Este resultado evidencia uma mudança local na precipitação.
Na Tabela 2, para o índice Precipitação Total Anual (PRCPTOT), observaram-se mudanças em 7 localidades, todas apontando um aumento da precipitação. Este resultado concorda com Santos e Brito (2007), que identificaram uma tendência positiva na precipitação para os Estados do Rio Grande do Norte e Paraíba, observando um aumento no percentual de dias chuvosos, e com os estudos de Haylock et al. (2006), que identificaram tendência de aumento da precipitação sobre a região Nordeste do Brasil. Observam-se ainda, da Tabela 2, para o índice PRCPTOT, tendências negativas em 4 localidades, porém, estas não são estatisticamente significantes e as magnitudes das tendências são inferiores aos seus erros padrões de estimativa. Portanto, não é seguro afirmar que estas tendências realmente estão ocorrendo. Para o número de dias com precipitação acima de $50 \mathrm{~mm}$ (R50mm), pode-se afirmar que ocorreram mudanças em apenas 3 localidades, todas mostrando aumento no número de dias com chuvas superiores a $50 \mathrm{~mm} / \mathrm{dia}$. Ressalta-se que em todas as localidades em que ocorreu aumento, também apresentaram aumento na quantidade de precipitação total anual, reforçando assim, as evidências de tendências positivas.

Investigando os índices, Dias Muito Úmidos (R95p) e Quantidade Máxima de Precipitação em 5 Dias Consecutivos (Rx5day) observou-se mudanças em 3 e 4 localidades, respectivamente. Nestes dois índices verificaram-se apenas tendências positivas, ou seja, mostrando que, em geral, ocorreu um aumento dos dias muito úmidos e das chuvas persistentes por até 5 dias consecutivos. Dos seis índices estudados, o PRCPTOT, R95p, R50mm e Rx5day apresentaram apenas tendências positivas, estatisticamente significantes. Para os dois

Tabela 1 - Índices climáticos dependentes da precipitação pluvial diária, com suas definições e unidades. O RR é o valor da precipitação diária. RR $\geq 1 \mathrm{~mm}$ representa um dia úmido e $\mathrm{RR}<1 \mathrm{~mm}$, um dia seco.

\begin{tabular}{|c|c|c|c|}
\hline ID & Nome do Indicador & Definição & Unidade \\
\hline DCS & Dias consecutivos secos & $\begin{array}{l}\text { Número máximo de dias } \\
\text { consecutivos com } \mathrm{RR}<1 \mathrm{~mm}\end{array}$ & dias \\
\hline DCU & Dias consecutivos úmidos & $\begin{array}{l}\text { Número máximo de dias } \\
\text { consecutivos com } R R>1 \mathrm{~mm}\end{array}$ & dias \\
\hline Rx5day & $\begin{array}{l}\text { Quantidade máxima de } \\
\text { precipitação em cinco dias }\end{array}$ & $\begin{array}{l}\text { Máximo anual de precipitação em } 5 \\
\text { dias consecutivos }\end{array}$ & $\mathrm{mm}$ \\
\hline R50mm & $\begin{array}{l}\text { Número de dias com } \\
\text { precipitação acima de } 50 \mathrm{~mm}\end{array}$ & $\begin{array}{l}\text { Número de dias em } 1 \text { ano em que a } \\
\text { precipitação foi }>50 \mathrm{~mm}\end{array}$ & dias \\
\hline R95p & Dias muito úmidos & $\begin{array}{l}\text { Precipitação anual total em que } \\
\text { RR }>95 \text { percentil }\end{array}$ & $\mathrm{mm}$ \\
\hline PRCPTOT & $\begin{array}{l}\text { Precipitação total anual nos } \\
\text { dias úmidos }\end{array}$ & $\begin{array}{l}\text { Precipitação total anual nos dias } \\
\text { úmidos (RR }>1 \mathrm{~mm})\end{array}$ & $\mathrm{mm}$ \\
\hline
\end{tabular}


Tabela 2: Tendências dos índices climáticos extremos dependentes da precipitação pluvial para 18 localidades distribuídas no Estado do Ceará. Os valores realçados em negrito apresentaram alta significância estatística $(p<0,05)$, os outros valores realçados apresentaram boa significância estatística $\mathrm{p}<0,1$.

\begin{tabular}{|c|c|c|c|c|c|c|c|c|c|}
\hline & & & & & & & & & \\
\hline Crato & $-7,23$ & $-39,40$ & 426 & $-0,143$ & 0,008 & 0,016 & $-0,011$ & $-0,786$ & 2,648 \\
\hline Fortaleza & $-3,73$ & $-38,57$ & 21 & 0,105 & 0,018 & 0,674 & 0,007 & 1,233 & 5,558 \\
\hline Iguatu & $-6,37$ & $-39,30$ & 217 & $-1,077$ & 0,023 & 0,221 & 0,007 & 0,819 & 4,497 \\
\hline Limoeiro do Norte & $-5,13$ & $-38,10$ & 30 & $-0,261$ & 0,012 & 0,006 & $-0,003$ & 0,191 & 0,680 \\
\hline Sobral & $-3,70$ & $-40,35$ & 69 & 0,127 & $-0,015$ & 0,190 & 0,016 & 1,778 & 2,953 \\
\hline Tauá & $-6,02$ & $-40,28$ & 402 & 0,140 & 0,035 & $-0,055$ & $-0,009$ & $-0,602$ & $-0,752$ \\
\hline Independência & $-5,45$ & $-40,48$ & 343 & $-0,312$ & 0,026 & 0,625 & 0,009 & 0,941 & 1,680 \\
\hline Assaré & $-6,87$ & $-39,87$ & 442 & $-0,544$ & 0,054 & 0,095 & $-0,010$ & $-0,440$ & 2,969 \\
\hline Nova Russas & $-4,73$ & $-40,57$ & 240 & 0,692 & $-0,031$ & 0,224 & 0,007 & 0,570 & 0,603 \\
\hline Canindé & $-4,35$ & $-39,30$ & 148 & $-0,042$ & 0,032 & $-0,059$ & 0,003 & 0,259 & 1,183 \\
\hline Iracema & $-5,82$ & $-38,30$ & 131 & $-0,216$ & 0,022 & $-0,030$ & $-0,011$ & $-0,218$ & 1,516 \\
\hline Quixeramobim & $-5,20$ & $-39,28$ & 191 & 0,495 & $-0,003$ & 0,030 & 0,003 & 0,073 & $-0,344$ \\
\hline Aracatí & $-4,57$ & $-37,77$ & 5 & 0,061 & $-0,021$ & $-0,446$ & $-0,002$ & $-0,441$ & $-1,453$ \\
\hline Brejo Santo & $-7,50$ & $-38,98$ & 381 & 0,160 & 0,029 & 0,372 & $-0,008$ & $-0,154$ & 0,919 \\
\hline Baturité & $-4,33$ & $-38,87$ & 175 & 1,512 & $-0,026$ & 0,039 & 0,001 & 0,111 & $-0,199$ \\
\hline Itapipoca & $-3,50$ & $-39,57$ & 108 & 0,602 & $-0,011$ & 0,233 & 0,003 & 0,427 & 0,027 \\
\hline Granja & $-3,13$ & $-40,83$ & 10 & 0,043 & $-0,010$ & 0,788 & 0,034 & 2,449 & 4,376 \\
\hline Viçosa do Ceará & $-3,57$ & $-41,08$ & 740 & 0,104 & 0,106 & 0,433 & 0,023 & 1,834 & 6,402 \\
\hline
\end{tabular}

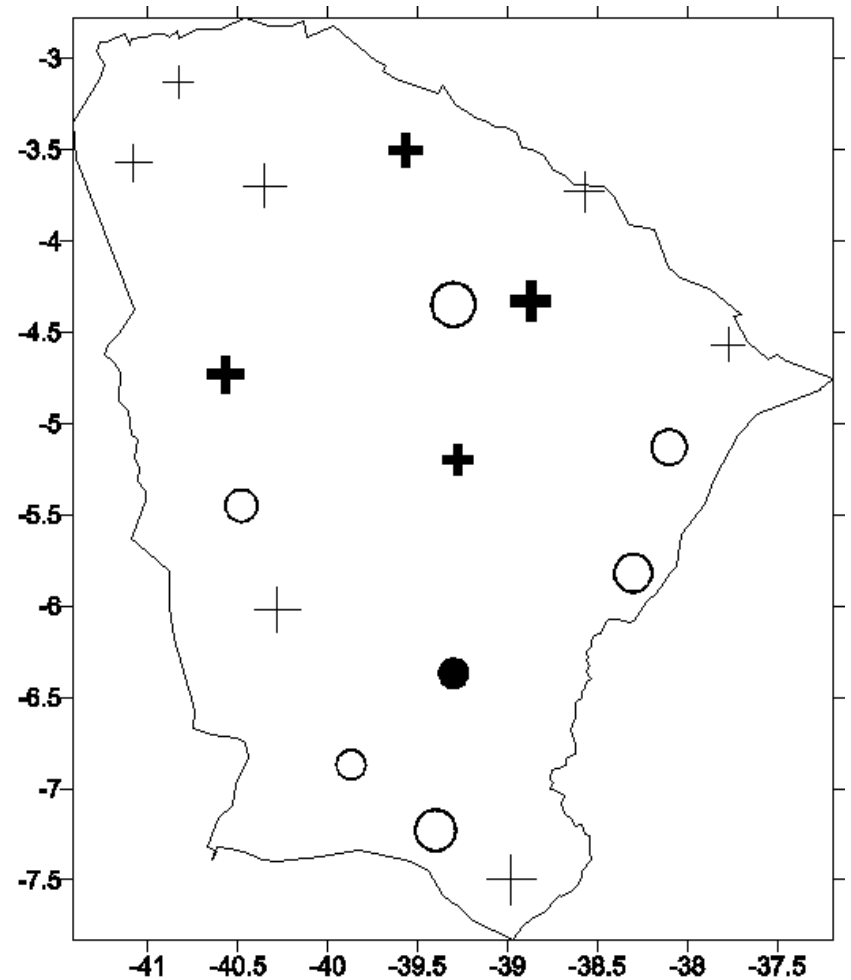

Figura 6 - Distribuição espacial das tendências do DCS, em dias/ ano.

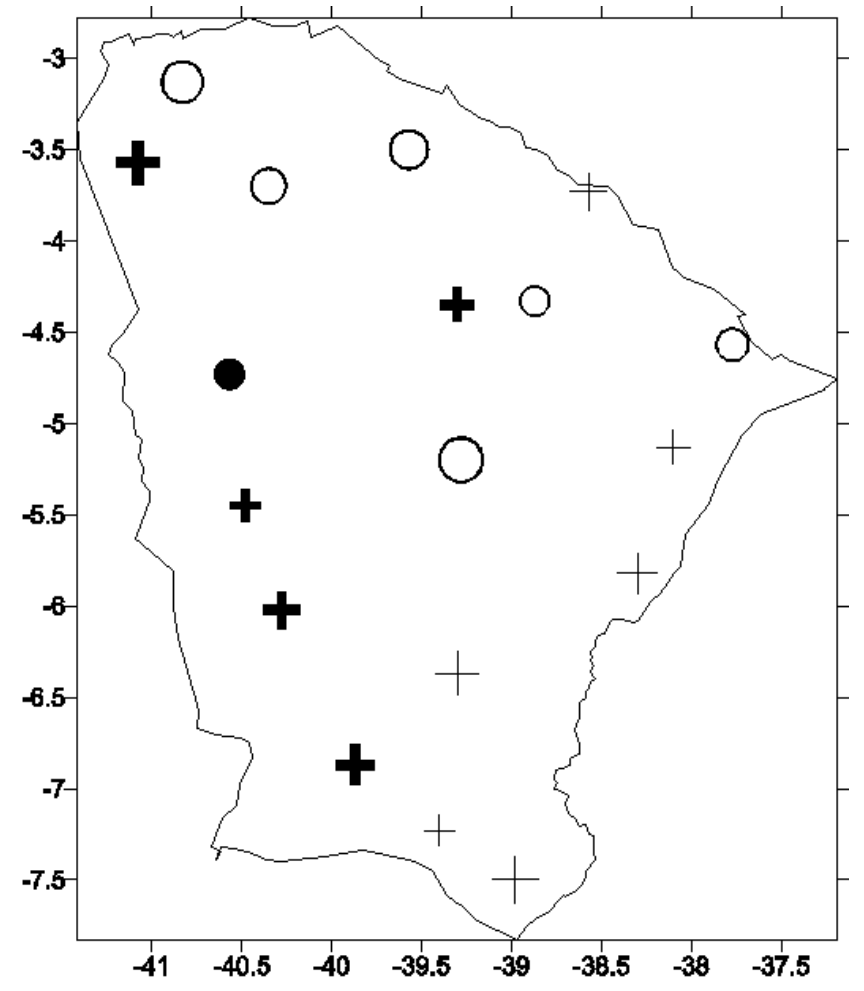

Figura 7 - Distribuição espacial das tendências do DCU, em dias/ ano. 
índices restantes, observaram-se tendências positivas e negativas (Tabela 2). Portanto, se faz necessário verificar a configuração da distribuição espacial de todos os índices para identificar as áreas em que ocorreram diminuição e aumento de cada índice.

$\mathrm{Na}$ Figura 6 observa-se a distribuição espacial das tendências do índice DCS, em dias/ano. Identificam-se tendências de valores positivos e negativos, mostrando comportamentos locais. No entanto, para os valores com significância estatística, observa-se que na região norte do Ceará há predominância no aumento dos dias consecutivos secos, assim como ocorre o oposto para o sul. Entretanto na distribuição espacial das tendências do DCU, em dias/ano (Figura 7), verifica-se predominância de tendências positivas sobre o Estado, com exceção de Nova Russas, localizada no meio oeste cearense. Vale salientar que, conforme descrito anteriormente, essa localidade foi a única em que os índices DCS e DCU apresentaram $\mathrm{p}<0,1$, evidenciando que nessa localidade o número de dias com chuva diminuiu.

A Figura 8 mostra a distribuição espacial das tendências do índice Rx5day, em mm/ano. Nota-se que houve predominância de tendência positiva, apresentando apenas 4 localidades com valores negativos, entretanto, sem significância estatística. Com relação às localidades com significância estatística, as

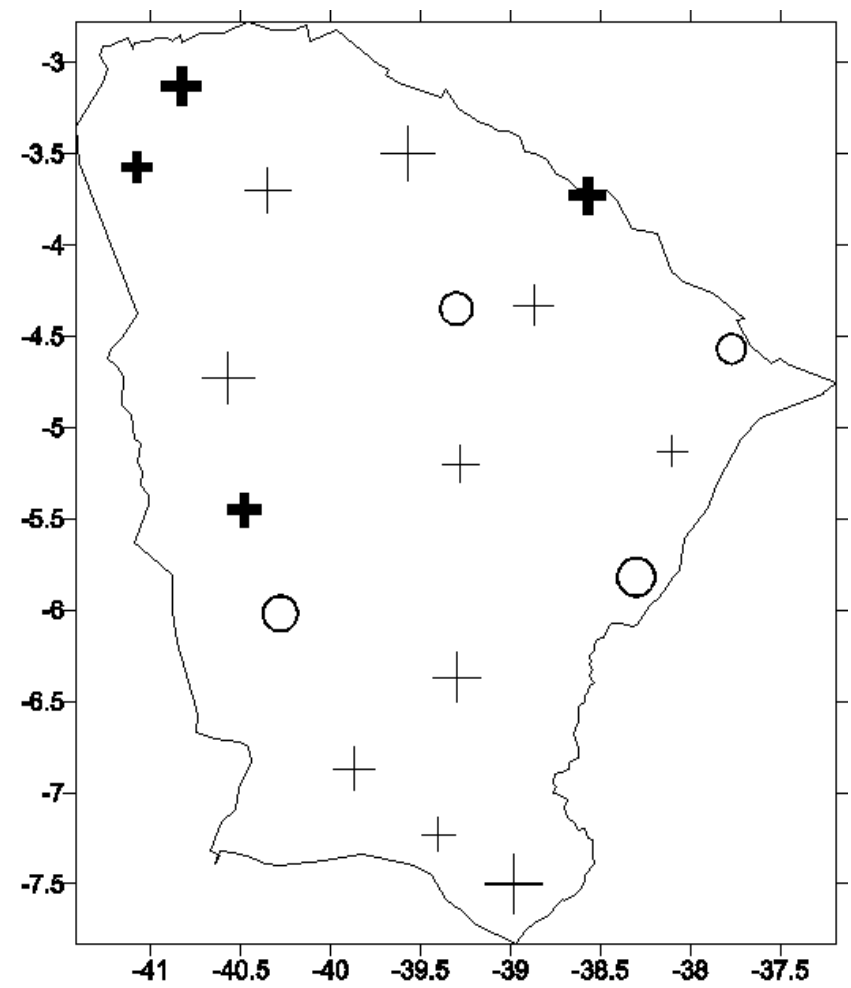

Figura 8 - Distribuição espacial das tendências do Rx5day, em $\mathrm{mm} / \mathrm{ano}$. mesmas estão distribuídas no norte e no meio oeste do estado. Este resultado mostra que no norte do Estado ocorreu aumento na precipitação em 5 dias consecutivos, tornando esses eventos chuvosos mais intensos, e chuvas intensas prolongadas podem causar grandes perdas econômicas.

Com relação à distribuição espacial das tendências do índice R50mm, em dias/ano (Figura 9), verifica-se um aumento no número de dias com chuvas acima de $50 \mathrm{~mm}$ no extremo norte do Ceará, esse comportamento se repete nas Figuras 10 e 11. Observa-se, portanto que o aumento no número de dias com chuva superior a $50 \mathrm{~mm}$ concorda com a elevação observada na precipitação total anual. A configuração espacial dos dias muito úmidos (R95p) é mostrada na Figura 10. Outra vez observa-se que, de um modo geral, há uma tendência de aumento de dias mais úmidos no norte do Ceará.

A Figura 11 apresenta a distribuição espacial das tendências do PRCPTOT (mm/ano). Observa-se uma tendência de aumento da precipitação sobre toda a área estudada. Tanto a região norte, quanto a sul apresenta tendências de aumento com significância estatística. A configuração da Figura 11 concorda com os estudos de Haylock et al. (2006) e Santos e Brito (2007), que também observaram tendência de aumento na precipitação total anual no NEB.

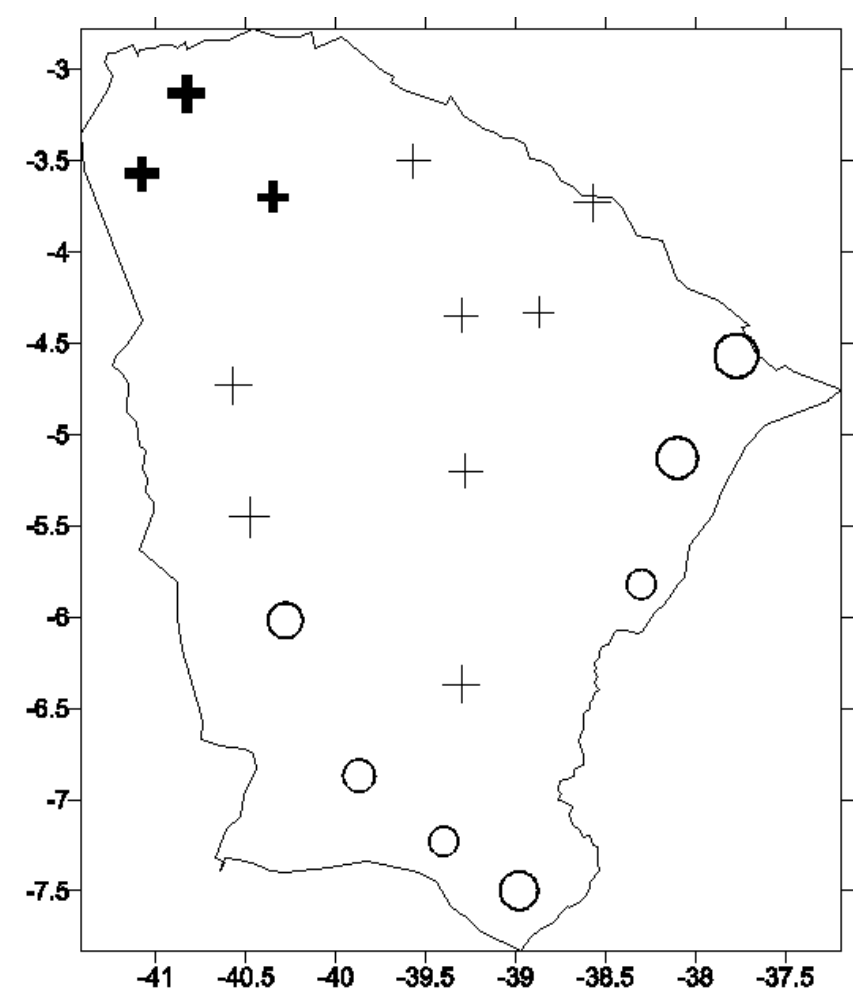

Figura 9 - Distribuição espacial das tendências do R50mm, em dias/ano. 
De forma geral, evidencia-se que os índices apontam para um aumento dos dias úmidos e da precipitação total anual, no entanto, não aponta uma diminuição dos dias consecutivos secos, o que caracteriza que está chovendo mais, em um menor número de dias. Estas características são preocupantes, pois se esse aumento de precipitação se mantiver, poderá acarretar inundação nos grandes centros, por exemplo do que ocorreu na cidade de Fortaleza, assim como a destruição de reservatórios e da agricultura pelo excesso de água em um curto intervalo de tempo.

\section{CONCLUSÕES}

Indicações de que o clima está mudando tem levado, recentemente, cientistas a estudar os extremos de tempo e clima. Neste contexto, este estudo apresenta uma análise das tendências de seis índices de precipitação anual para o Estado do Ceará.

De acordo com os resultados encontrados para os índices DCS e DCU, observou-se que ocorreram mudanças locais na precipitação. Os índices PRCPTOT, R50mm, R95p e Rx5day evidenciaram aumento em todas as localidades que apresentam significância estatística $(\mathrm{p}<0.1)$ (Tabela 2). Com base nesses resultados gerais, tem-se uma idéia de que houve um

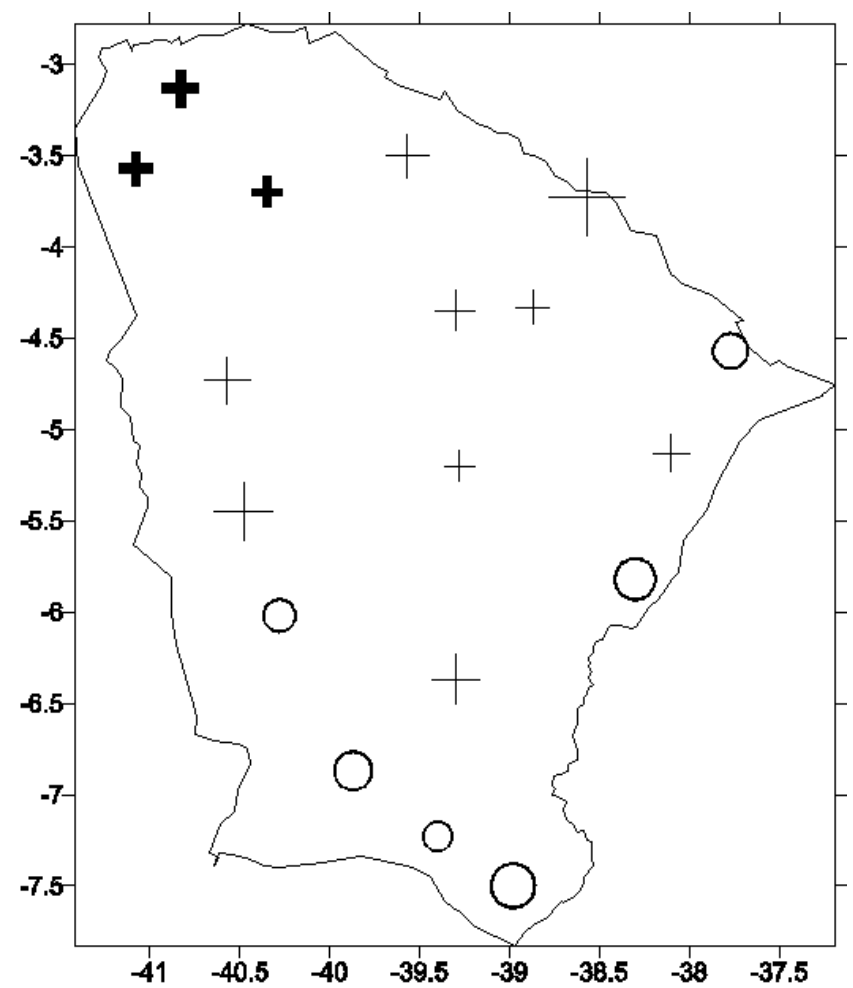

Figura 10 - Distribuição espacial das tendências do R95p, em mm/ ano. aumento nas condições de umidade sobre o Ceará. Analisando a distribuição espacial dos índices pode-se observar que o aumento de umidade deu-se predominantemente sobre a região norte do Estado. Entretanto, o índice PRCPTOT identificou também aumento na região sul do Ceará.

Finalmente, não se pode afirmar categoricamente, que as tendências observadas tenham ocorrido devido às mudanças globais do clima, mas a um somatório de fatores, tais como: desmatamento, mau uso do solo, entre outros. Também não é possível concluir que as tendências observadas estão mais ligadas às variações climáticas locais do que às globais, mas sim a uma soma destes fatores.

\section{AGRADECIMENTOS}

Os autores agradecem ao $\mathrm{CNPq}$ (Conselho Nacional de Desenvolvimento Científico e Tecnológico) pela concessão de bolsa para o primeiro autor; a FUNCEME (Fundação Cearense de Meteorologia) por fornecer os dados de precipitação, aos pesquisadores Xuebin Zhang e Feng Yang do Serviço de Meteorologia do Canadá, por disponibilizarem gratuitamente o software RClimdex 1.9.0, e aos revisores anônimos pelas valiosas sugestões propostas.

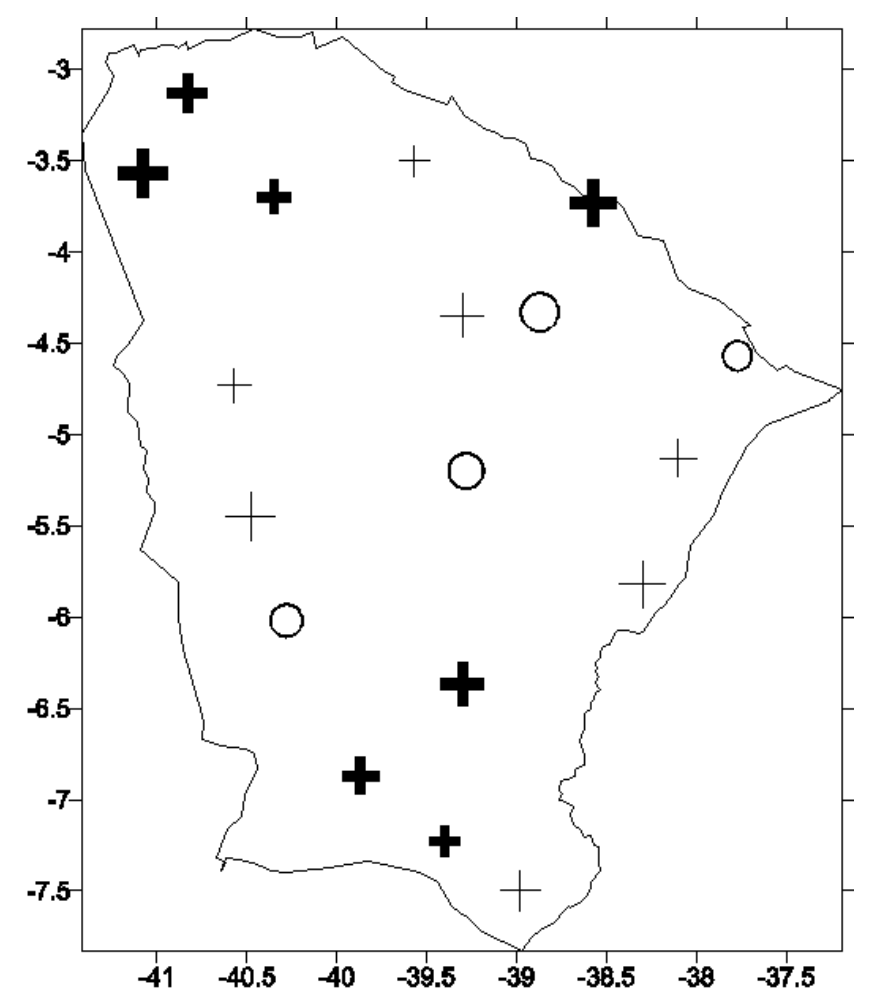

Figura 11 - Distribuição espacial das tendências da PRCPTOT, em $\mathrm{mm} / \mathrm{ano}$. 


\section{REFERÊNCIAS BIBLIOGRÁFICAS}

ALEXANDER, L. V., ZHANG, X., PETERSON, T. C., CAESAR, J., GLEASON, B., KLEIN TANK, A. M. G., HAYLOCK, M., COLLINS, D., TREWIN, B., RAHIMZADEH, F., TAGIPOUR, A., RUPA KUMAR, K., REVADEKAR, J., GRIFFITHS, G., VINCENT, L., STEPHENSON, D. B., BURN, J., AGUILAR, E., BRUNET, M., TAYLOR, M., NEW, M., ZHAI, P., RUSTICUCCI, M., VAZQUEZ-AGUIRRE, J. L. Global observed changes in daily climate extremes of temperature and precipitation. Journal of Geophysical Resources, 2006, doi: 10.1029=2005JD006290.

CANADIAN INTERNATIONALDEVELOPMENT AGENCY. RClimdex (0.9) User Manual, 25p., 2004.

HAYLOCK, M. R., PETERSON, T. C., ALVES, L. M., AMBRIZZI, T., ANUNCIAÇÃO, Y. M. T., BAEZ, J., BARROS, V. R., BERLATO, M. A., BIDEGAIN, M., CORONEL, G., GARCIA, V. J., GRIMM, A. M., KAROLY, D., MARENGO, J. A., MARINO, M. B., MONCUNILL, D. F., NECHET, D., QUINTANA, J., REBELLO, E., RUSTICUCCI, M., SANTOS, J. L., TREBEJO, I., VINCENT, L. A. Trends in total and extreme South American rainfall 1960-2000 and links with sea surface temperature. Journal of Climate, v. 19, p. 1490-1512, 2006.

HOOPER, D. U., CHAPIN, F. S., EWEL, J. J., HECTOR, E., INCHAUSTI, P., LAVOREL, S., LAWTON, J. H., LODGE, D. M., LOREAU, M., NAEEM, S., SCHMID, B., SETALA, H., SYMSTAD, A. J., VANDERMEER, J., WARDLE, D. A. Effects of biodiversity on ecosystem functioning: A consensus of current knowledge. Ecological Monographs, v. 75, p 3-35, 2005.

INTERGOVERNMENTAL PANEL ON CLIMATE CHANGE (IPCC). Climate Change 2007 - The Physical Science Basis. Contribution of Working Group I to the Fourth Assessment
Report of the IPCC. Cambridge Univ. Press, Cambridge, 2007.

KOSTOPOULO, E., JONES, P. D. Assesment of climate extremes in the Eastern Mediterrenean. Meteorology and Atmospheric Physics, v. 89, p. 69-85, 2005.

QIAN, W., LIN, X. Regional trends in recent precipitation indices in China. Meteorology and Atmospheric Physics, v. 90, p. 193-207, 2005.

SANTOS, C. A. C.; BRITO, J. I. B. Análise dos índices de extremos para o semi-árido do Brasil e suas relações com TSM e IVDN. Revista Brasileira de Meteorologia, v. 22, n. 3, p. 303-312, 2007.

SUBAK, S., PALUTIKOF, J. P., AGNEW, M. D., WATSON, S. J., BENTHAM, C. G., CANNELL, M. G. R., HULME, M., McNALLY, S., THORNES, J. E., WAUGHRAY, D., WOODS, J. C. The impact of the anomalous weather of 1995 on the UK economy. Climatic Change, v. 44, p. 1-26, 2000.

VINEY, N. R., BATES, B. C. It never rains on Sunday: The prevalence and implications of untagged multi-day rainfall accumulations in the Australian high quality data set. International Journal of Climatology, v. 24, p. 1171-1192, 2004.

WALSH, K., PITTOCK, A. B. Potential changes in tropical storms, hurricanes, and extreme rainfall events as a result of climate change. Climatic Change, v. 39, p. 199-213, 1998.

ZHANG, X., HEGERL, G., ZWIERS, F., KENYON, J. Avoiding inhomogeneity in percentile-based indices of temperature extremes. Journal of Climate, v. 18, p. 1641-1651, 2005.

ZHANG, X., YANG, F. RClimDex (1.0) User Guide. Climate Research Branch Environment Canada. Downsview (Ontario, Canada), 2004, 22p. 